\title{
IMPLEMENTACIÓN Y GENERALIZACIÓN DE SAAC EN CONTEXTOS DE EDUCACIÓN ESPECIAL
}

\author{
Ángela Segura Pérez \\ Universidad Católica de Valencia \\ ansepe2@mail.ucv.es \\ Claudia Tatiana Escorcia Mora \\ Universidad Católica de Valencia \\ claudia.escorcia@ucv.es
}

Fecha de Recepción: 12 Junio 2019

Fecha de Admisión: 25 Septiembre 2019

\section{RESUMEN}

El presente trabajo tiene como finalidad conocer el proceso mediante el que se desarrolla la implementación de Sistemas de comunicación alternativa y/ o aumentativa (SAACs) en el ámbito de los centros de educación especial (CEE). Para lograr este objetivo se realizó un análisis partiendo de una muestra de 13 docentes de Audición y Lenguaje en activo en CEE pertenecientes a la provincia de Valencia. Éste se realizó mediante una encuesta creada ad hoc. Tras el análisis comparativo se concluyó que la generalización del uso de estos sistemas presenta dificultades derivadas, principalmente, de la falta de coordinación y la baja participación de la familia. Se hace necesario, por tanto, propiciar espacios concretos para la coordinación y trabajo conjunto por parte de padres y profesionales en los diferentes entornos donde el alumno usuario de SAAC desarrolla su día a día. Todo ello perseguirá el objetivo de aumentar el grado de generalización de su uso, lo cual mejorará su desarrollo comunicativo-lingüístico y potenciará una correcta maduración psicosocial.

Palabras clave: dificultades en habla y lenguaje; necesidades educativas especiales; sistemas de comunicación alternativa y/o aumentativa (SAACs); escuela inclusiva.

\section{ABSTRACT}

AACS implementation and generalization in special education contexts. To achieve this aim, it was carryied out an analysis from a sample of 13 in-service hearing and speech teachers who are working in a special education school in the province of Valencia. Some different data has been obtained from a survey created ad hoc and these were contrasted with the existing bibliography. Following this comparative analysis it was concluded that the generalization of the use of these system is not adequated since there are difficulties that have mainly resulted from the lack of coordination, and also from the low involvement of the family. All of this has to be remedied through the collaborative work of the different environments where the student user of AAC develops their every- 


\section{IMPLEMENTACIÓN Y GENERALIZACIÓN DE SAAC EN CONTEXTOS DE EDUCACIÓN ESPECIAL}

day with the aim of increasing the degree of generalization which is going to improve their communicative linguistic decelopment and it is going to enhance a correct psycho-social maturity.

Keywords: speech and language difficulties; special educative needs; alternative and augmentative communication systems (AACS); inclusive school.

\section{INTRODUCCIÓN}

Los Sistemas Alternativos y/o Aumentativos de comunicación podrían definirse como una serie de recursos o estrategias dirigidos a facilitar tanto comprensión como expresión comunicativo-lingüística, con el objetivo de sustituir el habla (sistemas alternativos), o aumentarla (sistemas aumentativos) (Montero 2003). Por tanto, este tipo de sistemas serán de gran utilidad cuando el desarrollo comunicativo-lingüístico no se ha dado de forma correcta y existen graves dificultades en este ámbito.

Éstas pueden ser de tipología muy diversa pero, en los casos más graves, irán asociadas a una discapacidad. Además, se caracterizarán por limitar una correcta maduración psicoemocional (Tamarit, 1989) por lo que será importante ponerles solución de una forma lo más temprana posible con el fin de evitar problemas asociados.

En el ámbito escolar el alumnado con altas dificultades en este ámbito queda englobado en lo que se conoce como alumnado con Necesidades Educativas Especiales (en adelante alumnos con NEE o ANEE). La LOMCE en su artículo 73 sección 1 lo define como aquel "que requiera, por un periodo de su escolarización o a lo largo de toda ella, determinados apoyos y atenciones educativas específicas derivadas de discapacidad o trastornos graves de conducta" (p.38).

Todo este alumnado con dificultades en la comunicación y lenguaje y susceptible de ser usuario de SAAC trabajará con el especialista de Audición y Lenguaje (en adelante AyL) para mejorar sus competencias comunicativo-lingüísticas.

El docente de AyL desarrollará sus funciones tanto en centros educativos ordinarios como de educación especial (en adelante CEE). Es en estos en los que focalizaremos la presente investigación, pero: ¿Por qué abordar el tema de la comunicación alternativa y/o aumentativa en los CEE?

Fundamentalmente son tres los factores que nos empujan a ello:

El derecho y la necesidad de comunicar por parte de las personas usuarias de un SAAC.

La necesidad de formación sobre SAAC.

La importancia de las familias y entornos.

En relación a estos tres ejes cabe destacar la importancia que tiene para el alumnado con NEE el hecho de tener un modo de comunicarse y la repercusión de ello en su calidad de vida (Emperador-Garnace, 2018, Coronas y Basil, 2013 y O’Neill, Light,y Pope, 2018). Además, en este proceso será crucial que el docente de AyL cuente con una formación específica (Larraz, 2006). Por último, no se puede pasar por alto la gran importancia que tendrá la participación de la familia y entornos para lograr que el uso de estos sistemas de comunicación se torne funcional (EmperadorGarnace, 2018 y Ryan et al., 2018).

Visto todo esto resultará necesario conocer cómo se desarrollan los procesos de intervención y coordinación en el ámbito comunicativo-lingüístico dentro de los CEE con la finalidad de realizar ajustes enfocados en la generalización y uso de estos sistemas.

\section{OBJETIVOS DE LA INVESTIGACIÓN}

El objetivo principal de este trabajo es describir cómo interviene y coordina el docente de AyL la utilización de SAAC con el alumnado de CEE que requiere de su uso. Asimismo, interesa conocer la formación que poseen los docentes de AyL en SAAC y averiguar cómo se realiza la coordinación entre el docente de AyL y otros profesionales y familias. 


\section{PARTICIPANTES}

Se contó con la participación de un total de 13 docentes de Audición y Lenguaje que actualmente trabajan en centros de educación especial de carácter público en la provincia de Valencia. Tal como se observa en la Tabla 1 la muestra estuvo formada por 10 mujeres (un $76,92 \%$ de la muestra) y 3 hombres (un 23,08\%) de edades comprendidas entre los 26 y los 60 años de edad situándose la media en $(X=39,76)$. En cuanto a la experiencia docente estos valores se situaron entre 1 y 34 años siendo la media de $(X=11,54)$. Por último, destacar que de los 13 participantes 6 eran funcionarios de carrera $(46,15 \%), 6$ eran funcionarios en prácticas $(46.15 \%)$ y 1 funcionario interino $(7,7 \%)$.

Tabla 1

Características de los participantes

\begin{tabular}{lllll}
\hline participante & edad & sexo & años de experiencia & tipo de funcionario \\
\hline 1 & 40 & mujer & 15 & de carrera \\
2 & 60 & mujer & 34 & de carrera \\
3 & 54 & mujer & 21 & de carrera \\
4 & 44 & mujer & 10 & de carrera \\
5 & 31 & mujer & 2 & en prácticas \\
6 & 37 & hombre & 7 & interino \\
7 & 40 & mujer & 6 & en prácticas \\
8 & 44 & mujer & 16 & de carrera \\
9 & 30 & hombre & 6 & en prácticas \\
10 & 26 & mujer & 3 & en prácticas \\
11 & 32 & hombre & 1 & en prácticas \\
12 & 52 & Mujer & 28 & de carrera \\
13 & 27 & Mujer & 1 & en prácticas \\
\hline
\end{tabular}

\section{METODOLOGÍA E INSTRUMENTO}

\section{Metodología}

La realización del presente estudio se plantea bajo un paradigma de análisis cualitativo, más concretamente situado en la categoría de análisis cualitativo de tipo descriptivo, ya que la finalidad del mismo es la de describir la intervención y coordinación del docente de AyL en el ámbito comunicativo-lingüístico de los ANEE usuarios de SAAC. Para tal fin, se diseña una encuesta elaborada ad hoc. Ésta fue remitida a los diferentes CEE de la provincia de Valencia con la finalidad de acceder a la muestra y conseguir los datos necesarios. A lo largo de toda la investigación se tuvo en cuenta la ley de protección de datos y el código ético requerido para este tipo de investigaciones.

\section{Instrumento}

El instrumento de investigación elaborado para el estudio, se presenta en formato de encuesta online. Contiene 22 preguntas, tanto de respuesta cerrada, como de tipo abierto. Se encuentra dividida en cinco apartados:

Formación de los docentes de AyL en SAAC.

Perfil del alumnado usuario de SAAC.

Evaluación comunicativo-lingüística del alumnado.

Coordinación con respecto al uso de SAAC.

Generalización del SAAC.

En la primera parte de la encuesta, se explica la finalidad de la misma y se recogen los datos sociodemográficos de los participantes (edad, sexo, tipo de funcionario y años de experiencia como maestra/o de AyL). 
Cabe anotar que, en este manuscrito, sólo se presentan los resultados correspondientes a las preguntas relacionadas con la formación en SAAC del docente de AyL, así como a las referentes a la coordinación respecto al uso de SAAC.

\section{RESULTADOS ALCANZADOS}

Resultados en cuanto a formación en SAAC de los docentes de AyL.

Los datos sobre la formación y el tipo de SAAC en el que los docentes se han formado se muestran en las siguientes tablas:

Tabla 2

Formación específica en SAAC

\begin{tabular}{lll}
\hline ¿Ha recibido formación específica en SAAC? & $\mathrm{f}_{\mathrm{i}}$ & $\%$ \\
\hline sí & 12 & 92,31 \\
no & 1 & 7,69 \\
\hline
\end{tabular}

Tabla 3

Tipo de SAAC en los que se ha recibido formación

\begin{tabular}{llll}
\hline consideración & nombre & $\mathrm{f}_{\mathrm{i}}$ & $\%{ }^{*}$ \\
\hline SAAC & PECS & 10 & 76,92 \\
SAAC & Bimodal & 4 & 30,77 \\
SAAC & Benson schaeffer & 1 & 7,69 \\
SAAC & Apps & 6 & 46,15 \\
SAAC & Irisbond & 4 & 30,77 \\
SAAC & LSE & 2 & 15,38 \\
SAAC & Palabra complementada & 2 & 15,38 \\
otro & Apoyos ortofónicos & 3 & 23,08 \\
otro & Etran & 1 & 7,69 \\
otro & Pictogramas & 1 & 7,69 \\
\hline
\end{tabular}

* Porcentaje sobre total de la muestra

Como se puede observar en la tabla 2 casi el total de los encuestados se han formado específicamente en SAACs (únicamente uno de los docentes no ha realizado este tipo de formación). En cuanto a Ios SAACs escogidos para realizar esta formación destaca, por amplia mayoría el PECS seguido de las Apps.

\section{Resultados referentes a la coordinación.}

El análisis de estos resultados se ha realizado en relación a las familias y al resto de profesionales.

Coordinación con las familias:

Al hablar de la coordinación con las familias se analiza tanto el modo de coordinación como la frecuencia con la que se dan estas coordinaciones. 
Tabla 4

Modo de coordinación con las familias

\begin{tabular}{lll}
\hline tipo de coordinación & $\mathrm{f}_{\mathrm{i}}$ & $\% *$ \\
\hline reuniones & 9 & 69,23 \\
otros & 6 & 46,15 \\
sin determinar & 3 & 23,08 \\
\hline *Por
\end{tabular}

*Porcentaje sobre total de la muestra

En lo que respecta al modo de coordinarse con las familias una amplia mayoría de los docentes indicó que el medio más usual eran las reuniones. No obstante, también se destaca la existencia de otras vías de coordinación (mail, teléfono 0 agendas).

Tabla 5

Frecuencia de coordinación con las familias

\begin{tabular}{lll}
\hline Número de encuentros & $\mathrm{f}_{\mathrm{i}}$ & $\%{ }^{*}$ \\
\hline 2 al año & 1 & 7,69 \\
3 al año & 9 & 69,23 \\
4 al año & 1 & 7,69 \\
depende SAAC & 2 & 15,38 \\
depende evolución & 2 & 15,38 \\
demanda familia & 3 & 23,08 \\
no establecido & 1 & 7,69 \\
\hline *Porcentaje sobre total de la muestra
\end{tabular}

*Porcentaje sobre total de la muestra

Al hablar de la frecuencia de coordinación, se indica que los encuentros se realizan cada tres meses en la mayoría de los casos.

Coordinación con el resto de profesionales:

Al igual que en la coordinación con las familias, se debe tratar tanto el modo de coordinación que se realiza como la frecuencia.

Tabla 6

Modo de coordinación con el resto de profesionales

\begin{tabular}{lcc}
\hline tipo de coordinación & $\mathrm{f}_{\mathrm{i}}$ & $\%$ \\
\hline reunión & 6 & 46,15 \\
sin determinar & 7 & 53,85 \\
\hline
\end{tabular}

De estos resultados cabe destacar que en más de la mitad de los casos no se indicó el tipo de coordinación que se realizaba, siendo las reuniones, el único modo de coordinación indicado, las cuales se dieron en 6 de los 13 casos analizados. 


\section{IMPLEMENTACIÓN Y GENERALIZACIÓN DE SAAC EN CONTEXTOS DE EDUCACIÓN ESPECIAL}

Tabla 7

Frecuencia de coordinación con el resto de profesionales

\begin{tabular}{lll}
\hline frecuencia & $\mathrm{f}_{\mathrm{i}}$ & $\%{ }^{*}$ \\
\hline en cada sesión & 4 & 30,77 \\
semanalmente & 5 & 38,46 \\
trimestral & 1 & 7,69 \\
si es necesario & 6 & 46,15 \\
debería haber más & 1 & 7,69 \\
no establecido & 1 & 7,69 \\
\hline Porcentaje sobre total de la muestra
\end{tabular}

* Porcentaje sobre total de la muestra

En relación a la frecuencia de las coordinaciones con el resto de profesionales según los datos recogidos en la tabla 7 , suelen ser habituales al menos de forma semanal. No obstante, uno de los participantes indica que deberían darse con más frecuencia; es el caso del docente 13 que relata lo siguiente:

"Los momentos para la coordinación son breves y se dan cuando se va a recoger a un alumno, debería existir más tiempo de coordinación con los tutores y que esto se diera con frecuencia, puesto que la coordinación es fundamental tanto para la implementación del sistema como para su generalización.

Es de crucial importancia la coordinación al trabajo con el SAAC, pero desde el centro no se fomenta ni se da facilidad para poder tener tiempo dentro del horario de trabajo para que esto se pueda llevar a cabo de la mejor calidad posible, tanto con los profesionales como con las familias"

También aparece un grueso de docentes que indica que las coordinaciones se realizan sólo cuando es necesario, lo que parece indicar que éstas se realizan de forma algo arbitraria y sin corresponder a un plan específico de apoyo. Asimismo, los encuestados coinciden en afirmar que el tiempo disponible para estos encuentros es limitado y se hace normalmente en los momentos del cambio entre sesiones.

En nuestro estudio también se indagó acerca de la toma de decisiones en la implementación de un SAAC como parte de los procesos de coordinación. En la tabla 8 se muestran los resultados obtenidos:

Tabla 8

Toma de decisiones en relación al SAAC

\begin{tabular}{lll}
\hline ¿Quién Decide? & $\mathrm{f}_{\mathrm{i}}$ & $\% *$ \\
\hline AyL & 12 & 92,31 \\
Equipo educativo & 5 & 38,46 \\
Familia & 2 & 15,38 \\
Equipo psicopedagógico & 2 & 15,38 \\
Tutor & 3 & 23,08 \\
\hline
\end{tabular}

*Porcentaje sobre total de la muestra

Llama la atención dentro de estos resultados el gran peso que tiene el docente de AyL (92,32\%) a la hora de decidir qué tipo de SAAC será implementado, frente a la familia del alumno quien sólo decide en un escaso 15,38\%, cuando son ellas las que conviven con el usuario del SAAC y deben colaborar con su implementación. 


\section{DISCUSIÓN}

\section{Formación específica en SAAC}

Tal como se puede observar en el análisis de resultados casi la totalidad de docentes encuestados cuentan con formación específica en el ámbito de la comunicación alternativa y/ o aumentativa. Que un $92,31 \%$ de los participantes en el estudio haya decidido formarse en estos sistemas de comunicación, nos permite ver la gran necesidad que tiene el profesional de AyL de conocer métodos y formas de comunicación que ofrecer a sus alumnos.

Diversos autores indican que esta necesidad formativa puede deberse a que la formación inicial del docente de AyL no está totalmente relacionada con la realidad práctica posterior (Luque y Carrión, 2013 y Pegalajar, 2015). Además, cabe añadir que la intervención en SAAC requiere de unos aprendizajes precisos y concretos que variarán dependiendo del sistema a implementar. No obstante, algo básico y destacable para numerosos autores es la necesidad de que el docente de AyL se forme con la finalidad de poder capacitar a padres y otros profesionales en el ámbito comunicativolingüístico (Malik y Manaf, 2015) ya que, como se muestra en algunas investigaciones, los docentes con bagaje en este aspecto conseguirán una intervención con mayor porcentaje de éxito (Radici, Heboyan, Mantovani y De Leo, 2019).

La necesidad de formación específica de los docentes de AyL en el ámbito de los SAAC, también puede deberse a un sentimiento de incompetencia profesional frente a la realidad de las necesidades comunicativo-lingüísticas del alumnado con discapacidad (Chang, Early y Winton, 2005) lo que puede actuar como una barrera que afectará de forma directa al alumnado (Radici et al., 2019).

Queda claro pues que la formación específica es necesaria para la correcta intervención de estos docentes. Asimismo, en una época en la que la educación trata de realizar una transición hacia el paradigma de la inclusión esta formación se considerará clave (Monzón, 2011) y será necesario que se facilite mediante sistemas de formación continua o permanente (Luque y Carrión, 2013).

\section{Coordinación}

A la hora de plantearse el trabajo con un SAAC, la coordinación se convierte en un pilar fundamental. Diversos autores inciden en la importancia de la colaboración y coordinación entre familiares y profesionales para favorecer la implementación del sistema de comunicación (Hanline, Lindsay y Warren, 2018, Dabdub y Pineda, 2016 y Douglas, McNaughton y Light, 2013).

En la presente investigación se analiza la coordinación con dos grupos de agentes: las familias y el resto de profesionales que intervienen con el alumno.

\section{Coordinación con familias}

Como indican Smith y Hustad (2015) los padres están presentes en la mayor parte de los momentos de comunicación de sus hijos y, por tanto, se debe tener muy en cuenta a las familias en los procesos de implementación de un SAAC. En nuestro estudio hemos comprobado que la familia no es tenida muy en cuenta a la hora de decidir el tipo de SAAC a implementar. Resulta inquietante pensar que los profesionales, toman este tipo de decisiones alejados de las necesidades familiares, sin tener en cuenta sus fortalezas o limitaciones, con procesos poco coordinados. Algunos estudios desvelan que gran parte del éxito de un SAAC se basará en la colaboración y participación de los padres (Fortea-Sevilla et al., 2015 y García-Bacete, 2003). Sin embargo, en muchas ocasiones aparece una paradoja en la que, tal como indican lanni y Pérez (1998) "la escuela no encuentra a la familia cuando la convoca, a la vez que la familia no siempre tiene un lugar en la escuela" (p.132). Surge la duda entonces, de si este desencuentro se produce por no propiciar espacios de coordinación y comunicación efectivas entre escuela y familia, además de no permitir que la familia 
sea realmente participe de todos los procesos, relacionados con la implementación y uso de los SAAC, incluidos la evaluación, selección y uso de estos.

En nuestro estudio se observa que la coordinación con las familias suele darse en forma de reuniones (69,23\% de los encuestados) y con una periodicidad en su mayoría trimestral $(69,23 \%)$. Llegados a este punto cabe preguntarse: ¿Será este tipo de coordinación la más adecuada? ¿Es posible coordinarse con una reunión trimestral?

La investigación bibliográfica indica que, desde el inicio del trabajo con el SAAC, la familia es fundamental. Implementar un sistema de comunicación genera cambios en las dinámicas familiares por lo que estas deben ser tenidas muy en cuenta (Martínez et al., 1999 y Sánchez, 1988). Además, el contacto con padres y madres (o tutores legales) del alumnado implementado debe ser periódico, planificado y constante (Dabdub y Pineda, 2016 e lacono y Cameron, 2009) ya que las familias suelen precisar de guía, orientación e incluso formación (Stadskleiv, 2017 y Cabrera, Lizarzaro y Medina,2016, Douglas, McNaughton y Light, 2013).

En base a los resultados obtenidos en este estudio, parece claro que las coordinaciones llevadas a cabo por los docentes de la muestra de este estudio, resultarían algo escasas para lograr una cohesión escuela-familia (más específicamente docente de AyL-familia) lo que podría ver mermada la utilidad del SAAC (y, sobre todo, su generalización).

\section{Coordinación con profesionales}

El otro ámbito estudiado ha sido la coordinación con el resto de profesionales que atienden al alumno usuario de SAAC.

Según los datos recogidos estas coordinaciones se realizan con una frecuencia mayor que la coordinación con las familias. No obstante, en este caso gran parte de los participantes no indican el tipo de coordinación que se realiza mientras que, los que sí lo indican, apuntan a que se realizan reuniones (en un $46,15 \%$ de los casos).

La bibliografía consultada muestra, tal como sucede con la coordinación con las familias, la significación y necesidad de que esta se dé para poder realizar una implementación con garantías (Pinto, Simpson y Bakken, 2009). Añadido a esto se encuentran estudios que además de demandar una alta coordinación entre docentes y especialistas, abogan por una implementación conjunta en un ambiente lo más normalizado posible (Monfort, 2006). Se debe tener en cuenta también que este tipo de coordinación podría facilitar la formación del resto de profesionales (Martínez, Gracia, Latorre y González, 1999) y convertirse en clave para generar nuevas redes de apoyo (Tamarit, 2015) así como fomentar la sensibilización de todo el equipo docente. Por todo esto será importante que la coordinación del docente de AyL con el resto de profesionales sea dinámica, fluida y organizada.

Conocidos los resultados y comparados con la bibliografía disponible debe darse un tiempo a la reflexión. Según lo que se extrae de la comparativa realizada las coordinaciones en los CEE entre docentes de AyL y familias y entre AyL y profesionales distan de ser las necesarias para conseguir un uso funcional del SAAC implementado.

\section{CONCLUSIONES}

A modo de conclusión de este estudio cabe recordar que, tal como se ha observado, uno de los factores más importantes en relación a la implementación y generalización de los sistemas de comunicación alternativa y/o aumentativa es la coordinación y relación del docente de AyL con el resto de entornos donde se desenvuelve el alumno. Esto nos lleva a ver una clara necesidad de abrir las puertas del aula de Audición y Lenguaje ampliando el ámbito de intervención.

Por tanto, el docente de AyL debe ser conocedor de los beneficios que aporta una intervención colaborativa en el ámbito comunicativo-lingüístico. Ésta tendrá muy en cuenta a la familia, a la que 
se debe escuchar, atender y empoderar puesto que es el entorno donde el alumno pasa el grueso de su tiempo tal como indican García-Sánchez et al. (2014). Asimismo, una buena coordinación y comunicación hará posible que el resto de profesionales tomen conciencia de la utilidad del uso de estos sistemas de comunicación (Akpan, Lawrence y Beard, 2013).

La mejora en el ámbito de la coordinación logrará maximizar el éxito comunicativo-lingüístico que, tal como se ha visto, repercutirá en el desarrollo holístico de cada alumno. También se favorecerá la unión del micro-meso y macrosistema donde éste se desenvuelve (Escorcia, 2019). Ambos factores repercutirán en un aumento de la calidad de vida (Escribano y Tamarit, 2019, y Hill, 2006).

Para finalizar solo queda hacer hincapié en la importancia de la comunicación, eje sobre el que gira la presente investigación. Tal y como indican Martínez et al. (1999):

Para poder intervenir de forma precoz y eficiente en el desarrollo de la comunicación en personas con discapacidad, es preciso tomar como premisa fundamental el hecho de que todo ser humano quiere comunicarse, y es preciso darle oportunidades y medios apropiados para hacerlo. (p.650)

\section{REFERENCIAS BIBLIOGRÁFICAS}

Akpan, J. P., \& Beard, L. A. (2013). Overview of assistive technology possibilities for teachers to enhance academic outcomes of all students. Universal Journal of Educational Research, 1(2), 113-118.

Chang, F., Early, D. M., \& Winton, P. J. (2005). Early Childhood Teacher Preparation in Special Education at 2- and 4-Year Institutions of Higher Education. Journal of Early Intervention, 27(2), 110-124. https://doi.org/10.1177/105381510502700206

Coronas Puig-Pallarols, M., \& Basil Almirall, C. (2013). Comunicación aumentativa y alternativa para personas con afasia. Revista de Logopedia, Foniatría y Audiología, 33(3), 126-135. https://doi.org/10.1016/j.rlfa.2012.10.004

Dabdub, M., \& Pineda, A. (2016). La atención de las necesidades educativas especiales y la labor docente en la escuela primaria. Revista Costarricense de Psicologia, 34(1), 42.

Douglas, S. N., McNaughton, D., \& Light, J. (2013). Online Training for Paraeducators to Support the Communication of Young Children. Journal of Early Intervention, 35(3), 223-242. https://doi.org/10.1177/1053815114526782

Emeperador- Garnace, X. R. (2018). The Interrelationship of Language And The Brain And Language Disorders In Augmentative And Alternative Communication (AAC): Findings From A Systematic Review Of The Literature. PEOPLE: International Journal of Social Sciences, 3(3).

Escorcia, C. (2019). Evolución, bases conceptuales y presente de la atención temprana. En: C. Escorcia Mora y L. Rodríguez García, ed., Prácticas de Atención Temprana Centradas en la Familia y en Entornos Naturales. Madrid: UNED, pp.3-32.

Escribano, L. y Tamarit, J. (2019). Calidad de vida familiar en el día a día. En: C. Escorcia y L. Rodríguez, Prácticas de Atención Temprana Centradas en la Familia y en Entornos Naturales. Madrid: UNED, pp.147-161.

García-Bacete, F.-J. (2003). Las relaciones escuela-familia: Un reto educativo. Infancia y Aprendizaje, 26(4), 425-437. https://doi.org/10.1174/021037003322553824

García-Sánchez, F. A., Escorcia Mora, C. T., Sánchez-López, M. C., Orcajada Sánchez, N. y Hernández-Pérez, E. (2014). Atención Temprana centrada en la familia. SIGLOCERO Revista Española sobre Discapacidad Intelectual, 45 (3), 6-27.

Hanline, M. F., Lindsay R., \& Warren, A. W. (2018). The Outcomes of Professional Development on AAC Use in Preschool Classrooms: A Qualitative Investigation. Infants \& Young Children, 31(3), 231-245. 
Hill, K. (2006). A Case Study Model for Augmentative and Alternative Communication Outcomes. Assistive Technology Outcomes and Benefits, 3(1), 53-66.

Iacono, T., \& Cameron, M. (2009). Australian Speech-Language Pathologists' Perceptions and Experiences of Augmentative and Alternative Communication in Early Childhood Intervention. Augmentative and Alternative Communication, 25(4), 236-249. https://doi.org/10.3109/07434610903322151

Ianni, N. D., \& Pérez, E. (1998). La convivencia en la escuela, un hecho, una construccion: hacia una modalidad diferente en el campo de la prevención.

Larraz, C. (2006). Comunicación Aumentativa. Minusval, Dossier de comunicación aumentativa y alternativa, 17-19.

Ley Orgánica 8/2013, de 9 de diciembre, para la mejora de la calidad educativa.Boletín Oficial del Estado, 295, de 10 de diciembre de 2013, 97858 a 97921.

Luque de la Rosa, A., \& Carrión Martínez, J. J. (2013). El maestro de audición y lenguaje: Formación, experiencia previa y acceso. Revista Electrónica Interuniversitaria de Formación del Profesorado, 16(2), 39-54. https://doi.org/10.6018/reifop.16.2.181141

Malik, S., \& Manaf, U. K. B. A. (2015). Role of special education curriculum in adjustment of physically handicapped children in society. PEOPLE: International Journal of Social Sciences, 1(1).

Martínez, A. B., Gracia, C., Latorre, A., \& González, R. (1999). Proceso de implantación y generalización de sistemas alternativos y aumentativos de comunicación (SAAC) en un centro de educación especial. En Hacia una nueva concepción de la discapacidad: Actas de las III Jornadas Científicas de Investigación sobre personas con discapacidad (pp. 639-652). Amarú.

Monfort, M. (2006). La comunicación bimodal: una ayuda para el desarrollo del lenguaje y de la comunicación. Madrid: Asociación Alanda

Montero, P. (2003). Sistemas alternativos y aumentativos de comunicación (SAAC) y accesibilidad: Bases teóricas de los SAAC. Puertas a la lectura, (4), 129-136.

Monzón, J. (2011). Asesoramiento hacia la escuela inclusiva y formación en centros. Revista interuniversitaria de formación del profesorado, (70), 111-126.

O'Neill, T., Light, J., \& Pope, L. (2018). Effects of Interventions That Include Aided Augmentative and Alternative Communication Input on the Communication of Individuals with Complex Communication Needs: A Meta-Analysis. Journal of Speech, Language, and Hearing Research, 61(7), 1743-1765. https://doi.org/10.1044/2018 JSLHR-L-17-0132

Pinto, P., Simpson, C., \& Bakken, J. P. (2009). Research-based instructions to increase communication skills for students with severe disabilities. International journal of special education, 24(3), 11.

Radici, E., Heboyan, V., Mantovani, F., \& De Leo, G. (2019). Teachers' Attitudes Towards Children Who Use AAC in Italian Primary Schools. International Journal of Disability, Development and Education, 66(3), 284-297. https://doi.org/10.1080/1034912X.2018.1495321

Ryan, S. E., Shepherd, T. A., Renzoni, A. M., Servais, M., Kingsnorth, S., Laskey, C., ... Bradley, K. (2018). Responsiveness of a parent-reported outcome measure to evaluate AAC interventions for children and youth with complex communication needs. Augmentative and Alternative Communication, 34(4), 348-358. https://doi.org/10.1080/07434618.2018.1520296

Sánchez, I. (1998). Otra intervención curricular comunicativa: Ios SAC. Campo Abierto. Revista de Educación, 15(1), 173-211.

Smith, A. L., \& Hustad, K. C. (2015). AAC and Early Intervention for Children with Cerebral Palsy: Parent Perceptions and Child Risk Factors. Augmentative and Alternative Communication, 31(4), 336-350. https://doi.org/10.3109/07434618.2015.1084373 
Stadskleiv, K. (2017). Experiences from a support group for families of preschool children in the expressive AAC user group. Augmentative and Alternative Communication, 33(1), 3-13. https://doi.org/10.1080/07434618.2016.1276960

Tamarit, J. (1989). Uso y abuso de los sistemas alternativos de comunicación. Comunicación, Lenguaje y Educación, 1(1), 81-94. https://doi.org/10.1080/02147033.1989.10820868

Tamarit, J. (2015). La transformación de los servicios hacia la calidad de vida. Una iniciativa de innovación social de FEAPS. Siglo Cero. Revista Española sobre Discapacidad Intelectual, 46(3), 47. https://doi.org/10.14201/scero20154634771 
